INPLASY

PROTOCOL

To cite: Zhang et al. Risk prediction models for intensive care unit-acquired weakness in intensive care unit patients: $A$ systematic review. Inplasy protocol 202140010. doi: 10.37766/inplasy2021.4.0010

Received: 01 April 2021

Published: 02 April 2021

Corresponding author: Wei Zhang

1799012058@qq.com

Author Affiliation: The First Affiliated Hospital of Wannan Medical College

Support: $201904 a 07020034$.

Review Stage at time of this submission: Preliminary searches.

Conflicts of interest: None declared.

\section{Risk prediction models for intensive care unit-acquired weakness in intensive care unit patients: A systematic review}

\author{
Zhang, W1; Tang, Y2; Liu, H3; Yuan, LP4.
}

Review question / Objective: In this systematic review, we aimed to systematically summarize and compare the different prediction models of ICU acquried weakness (ICU-AW) based on their respective basic characteristics, the methods used to develop them, their methodological quality, their prediction performance, and the predictors considered in the models.

Condition being studied: Investigate and analyze the risk factors of ICU-AW that may occur in ICU patients. Based on MRC scores as the diagnostic basis, and use the identified risk factors as predictors to develop and verify the risk prediction model of ICU-AW.

INPLASY registration number: This protocol was registered with the International Platform of Registered Systematic Review and Meta-Analysis Protocols (INPLASY) on 02 April 2021 and was last updated on 02 April 2021 (registration number INPLASY202140010).

\section{INTRODUCTION}

Review question / Objective: In this systematic review, we aimed to systematically summarize and compare the different prediction models of ICU acquried weakness (ICU-AW) based on their respective basic characteristics, the methods used to develop them, their methodological quality, their prediction 
performance, and the predictors considered in the models.

Condition being studied: Investigate and analyze the risk factors of ICU-AW that may occur in ICU patients. Based on MRC scores as the diagnostic basis, and use the identified risk factors as predictors to develop and verify the risk prediction model of ICU-AW.

\section{METHODS}

Participant or population: Adult critically ill patients who are admitted to an ICU.

Intervention: Not applicable.

Comparator: Not applicable.

Study designs to be included: Cohort studies and case-control studies.

Eligibility criteria: (1) Studies that regarded ICU patients older than 18 years old and in which patients had not been excluded on the basis of their race, nationality, or course of illness; (2) studies that involved the development of an ICU-AW risk prediction model for ICU patients and specific explanation of the tools used to diagnose ICU-AW and the main evaluation methods and steps; (3) studies that involved the internal and/or external verification of a prediction model after it had been developed.

Information sources: Four Englishlanguage databases (PubMed, Embase, Cochrane Library, Scopus) and three Chinese-language databases (China National Knowledge Infrastructure, Weipu, and WanFang databases) were searched to collect studies regarding ICU-AW risk prediction models for ICU patients. In addition, the reference lists of the included studies were searched to supplement the acquisition of relevant literature.

Main outcome(s): 1. The general characteristics of the different prediction models; 2. The method of development and verification of the prediction models; 3 . The performance and applicability of the model;
4. The predictive factors considered in the model.

Quality assessment / Risk of bias analysis: Two reviewers independently evaluated the quality of the included studies according to the Newcastle-Ottawa Scale and evaluated the risk of bias and applicability of the models using the Prediction model Risk of Bias Assessment Tool.

Strategy of data synthesis: Descriptive analysis methods were used to summarize the data.

Subgroup analysis: Not applicable.

Sensitivity analysis: Not applicable.

Language: The search was restricted to English- or Chinese- language studies.

Country(ies) involved: China.

Keywords: Intensive care; ICU-acquired weakness; risk prediction; model.

Contributions of each author:

Author 1 - Wei Zhang.

Author 2 - Yun Tang.

Author 3 - Huan Liu.

Author 4 - Li ping Yuan. 